\title{
Pressure natriuresis and diuresis are differentially regulated depending on age and sex
}

\author{
Yang Gyun Kim, Ju-Young Moon, Sang Ho Lee
}

Department of Internal Medicine-Nephrology, Kyung Hee University School of Medicine, Seoul, Korea

Background: The renal capacity for handling salt and water is linked to hypertension. This study aimed to clarify the sex- and age-related natriuretic and diuretic differences that occur in blood pressure (BP) regulation.

Methods: We analyzed two datasets: one representing 235 patients with nondiabetic chronic kidney disease from the E-SPECIAL trial, which evaluated the effect of a low-salt diet (LSD) on olmesartan for lowering albuminuria, and one representing 4,937 participants enrolled in the Korean Genome and Epidemiology Study (KoGES).

Results: In the E-SPECIAL trial, BP was lower in premenopausal women (PRE) than in younger men (YM), whereas no BP difference was evident between postmenopausal women (POST) and older men (OLD). LSD with olmesartan decreased urine sodium in YM, POST, and OLD but not PRE. A positive correlation between BP and urine sodium was observed in only the younger groups (PRE, YM). Urine volume was greater in PRE than in YM, and urine concentration was reciprocally lower in PRE than in YM. Urine volume was positively correlated with BP in PRE but negatively associated with BP in YM. Urine volume and urine sodium were the most decisive predictors of BP in PRE. In the KoGES, BP was lowest in PRE. Urine sodium was higher in PRE than POST, whereas it was higher in OLD than in YM. The correlation between BP and urine sodium was augmented in the younger groups. Conclusions: Pressure-natriuretic and pressure-diuretic responses were well conserved in PRE and mitigated in POST. Augmented natriuresis and diuresis might contribute to lower BP in PRE.

Corresponding author: Yang Gyun Kim

E-mail: apple8840@hanmail.net 